\title{
Calibration methods of new device for human blood pressure measurement
}

\author{
Eukasz Stala ${ }^{1 *}$, Krzysztof Tomczuk ${ }^{1}$ \\ ${ }^{1}$ Wroclaw University of Science and Technology, Faculty of Mechanical and Power Engineering, \\ Department of Criogenics, Aeronautics and Process Engineering, 50-370 Wroclaw, Wybrzeze \\ Wyspianskiego 27, Poland
}

\begin{abstract}
The paper presents two methods of determining calibration curve of a new device for blood pressure measurement. The device was developed at Wrocław University of Science and Technology. First method is based on parallel measurement of systolic and diastolic pressure measurement with use of reference device such as sphygmomanometer and researched new device with pneumatic sensor equipped with voltage type output. Obtained data (systolic $\mathrm{p}_{\mathrm{s}}$ and diastolic $\mathrm{p}_{\mathrm{d}}$ pressure, maximum $\mathrm{u}_{\mathrm{s}}$ and minimum $\mathrm{u}_{\mathrm{d}}$ voltage) was then used to determine individual pressure-voltage characteristic of the device, which can be represented as a linear equation. Second method is based on substitution of experimentally proved coefficient $\mathrm{b}$ with its analytical equivalent extracted from mathematical model of described pneumatic sensor. Described methods were verified experimentally and compared. Metrological parameters of the device were designated.
\end{abstract}

\section{Introduction}

Blood pressure measurement is a basic element of medical examination. Depending on the case different method is used. The most accurate, invasive method, is used mostly for critical care and operating room measurements. This method can be also used for the blood pressure waveform recording [1]. Less accurate but considered as a golden standard [2] among the non-invasive methods for blood pressure measurement is auscultatory method. In this method, with use of sphygmomanometer and stethoscope, Korotkoff sounds (first and fourth phase [1]) can be registered and distinguish systolic and diastolic pressure. Auscultatory method is widely used in clinical measurements [2]. Another non-invasive measurement method is oscillometric method. The method uses sphygmomanometer cuff with eletronic pressure sensor which transduces voltage reading to pressure basing on calibration equation [3]. Different oscillometric devices use various algorithms and calibration coefficients which leads to differing blood pressure readings [3]. This method is not considered very accurate, however it is popular for domestic uses [4]. Both mentioned methods use cuff which can be one of measurement disruption sources [1]. Auscultatory and oscillometric methods cannot register blood pressure waveforms, which currently leaves invasive method as the only option for this task. The device developed at Wrocław University of Science and Technology, as well as proposed calibration methods, do not rely on cuff. Due to this fact, one of the error sources is eliminated. A new measurement method with use of the newly developed device also allows to examine the blood pressure waveform [5], which can be a source of important data for general practice and clinical diagnosis. Even though current build does not allow the continous monitoring of the blood pressure waveform, the

* Corresponding author: lukaszstala2@gmail.com" 
ability to aquire the blood pressure waveform scaled in pressure units with noninvasive method is a novelity.

\section{Measuring device}

Due to the lack of non-invasive accurate method for pressure measurement and blood pressure waveform recording, the new device was developed. The optimised build_would be ideal for clinical uses as well as for personal use for patients with cardiovascular problems. The device consists of a personal computer (3) and a pneumatic sensor (1) connected to an electronic-pneumatic system (2) by pneumatic wire, as seen on Figure 1.
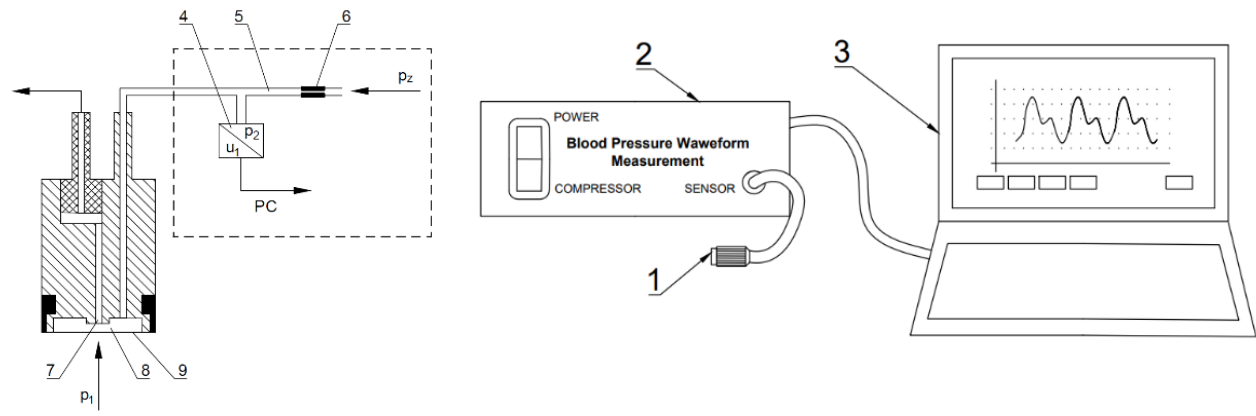

Fig. 1. Scheme of nozzle and electronic-pneumatic system (left) and device setup (right). (4 pressure-voltage transducer, 5 - pneumatic wire, 6 - throttle, 7 - vent nozzle, 8 - measurement chamber, 9 - membrane, $\mathrm{p}_{\mathrm{z}}$ - supplied pressure, $\mathrm{p}_{1}$ - vessel pressure, $\mathrm{p}_{2}$ - measured pressure). [7]

The device was previously tested in laboratory conditions with use of an artificial artery. The artery imitation was built using soft rubber plate with groove covered by a thin latex membrane. While the soft tissue surrounding the blood vessel was simulated with rubber plate, the membrane and groove were equivalent of the artery. After completing the laboratory setup the test was performed. The pneumatic generator supplied the pressure to the groove simulating desired blood pressure waveform (during the dynamic flow changes). The input and output pressures were measured with manometers and recorded with transducers on a digital oscilloscope [5]. Results of this experiment proved that the device static characteristic is linear [5] and has excellent dynamic parameters just as presented on Figure 2.

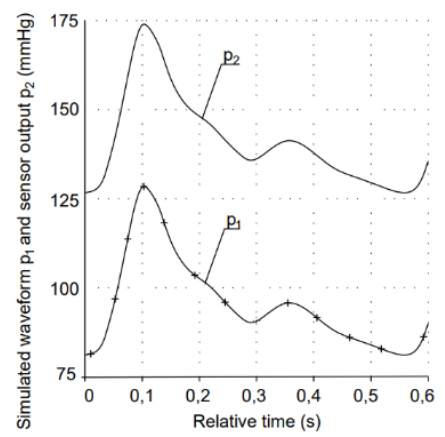

Fig. 2. Comparison of pressure inside the artery imitation $\left(\mathrm{p}_{1}\right)$ and device's readings $\left(\mathrm{p}_{2}\right), \mathrm{x}-$ pressure calculated based on static characteristic. [5]

The experiment was the starting point to optimisation and improvement of newly developed device, since the measurements performed on volunteers were less accurate than those performed in laboratory conditions on the artificial artery. It was assumed that the 
difference had its source in the geometric dimensions of human and artificial artery and current built of the device had to be calibrated, since the bare transducer equation provided by the manufacturer was insufficiently accurate.

\section{Experimental}

The measurements for preliminary calibration purposes were conducted on 14 volunteers ( 7 female volunteers, 7 male volunteers) in age between 20 and 40 years old in accordance to International Protocol for Validation of Blood Pressure Measuring Devices in Adults [6]. Before taking measurements the volunteers were asked to rest about 5 minutes for the blood flow stabilisation. The measurements were taken with use of auscultatory method (the chosen devices were ERKA Perfect-Aneroid Sphygmomanometer and Erka Precise stethoscope). After the auscultatory method was performed the new method was used as described below.

The measurement of blood pressure proceeded as follows: the sensor's nozzle is pressed directly against patient's radial artery or any other easily accessible artery (located directly under the skin), at the same time the processed signal is displayed as the blood pressure waveform on the designed user interface on the PC screen. During the measurement process the amplitudes of waveforms increase at first to then decrease slightly. Designed application selects the waveform with highest amplitude which designates systolic and diastolic pressure and displays all accessible parameters associated with cardiovascular condition of a patient.

\section{Calibration methods}

The calibration was performed based on data extracted during the experiment. The data sets of each volunteer consisted of diastolic $\left(\mathrm{p}_{\mathrm{d}}\right)$ and systolic $\left(\mathrm{p}_{\mathrm{s}}\right)$ pressure $[\mathrm{mmHg}]$ from auscultatory method as well as diastolic $\left(\mathrm{U}_{\mathrm{d}}\right)$ and systolic $\left(\mathrm{U}_{\mathrm{s}}\right)$ voltage values $[\mathrm{V}]$ from new device measurements. The data sets were then randomly divided in two groups - first for calibration purposes (10 sets) and second for calibration validation (4 sets).

The first calibration method (Method 1) based on device's linear static characteristic (Paragraph 2.) allowed to extract linear equation's coefficients from 10 data sets as seen in Table 1.

Table 1. Obtained data sets and calculated coefficients.

\begin{tabular}{|c|c|c|c|c|c|c|}
\hline \multirow{3}{*}{$\begin{array}{c}\text { Set } \\
\text { number }\end{array}$} & \multicolumn{2}{|c|}{ New Device } & \multicolumn{2}{|c|}{ Auscultatory method } & \multicolumn{2}{|c|}{ Coefficients } \\
\hline & $\mathrm{U}_{\mathrm{d}}$ & $\mathrm{U}_{\mathrm{s}}$ & $\mathrm{p}_{\mathrm{d}}$ & $\mathrm{p}_{\mathrm{s}}$ & $\mathrm{a}$ & $\mathrm{b}$ \\
\hline & \multicolumn{2}{|c|}{ [V] } & \multicolumn{2}{|c|}{$[\mathrm{mmHg}]$} & \multicolumn{2}{|c|}{$[\mathrm{mmHg}]$} \\
\hline 1. & 1.05 & 1.47 & 65 & 120 & -73 & 131 \\
\hline 2. & 1.02 & 1.53 & 55 & 96 & -27 & 80 \\
\hline 3. & 1.02 & 1.37 & 60 & 100 & -57 & 114 \\
\hline 4. & 1.04 & 1.35 & 58 & 97 & -73 & 126 \\
\hline 5. & 1.01 & 1.36 & 77 & 123 & -56 & 131 \\
\hline 6. & 1.26 & 1.68 & 78 & 117 & -39 & 93 \\
\hline 7. & 1.17 & 1.61 & 79 & 125 & -43 & 105 \\
\hline 8. & 1.10 & 1.51 & 70 & 120 & -64 & 122 \\
\hline 9. & 1.10 & 1.52 & 71 & 120 & -57 & 117 \\
\hline 10. & 1.34 & 1.65 & 66 & 94 & -55 & 90 \\
\hline
\end{tabular}


Then the mean $a$ and $b$ coefficients were calculated and substituted to the general calibration equation (Equation 1.).

$$
p_{x}=a+u_{x} \cdot b
$$

Alternatively the second calibration method (Method 2) was also performed. The $b$ coefficient of general calibration equation was calculated based on $k$-factors of transducer and sensor in accordance to Equation 2. Transducer's $k$-factor is calculated using Equation 3, where $A_{l}$ is the area of membrane on the external side of measurement chamber's membrane and $A_{2}$ is the area of membrane on the internal side of the measurement chamber's membrane. Sensor's $k$-factor is calculated using Equation 4 , where $\Delta u$ is the increment of transducer's voltage and $\Delta p_{2}$ is the increment of pressure inside the measuring chamber [5].

$$
\begin{gathered}
b=\frac{1}{k_{1} \times k_{2}} \\
k_{1}=\frac{A_{1}}{A_{2}} \\
k_{2}=\frac{\Delta u}{\Delta p_{2}}
\end{gathered}
$$

This procedure led to the designation of two different calibration equations. Equation 5 corresponds to standard calibration method. Equation 6 corresponds to the second calibration method where coefficient $b$ was calculated.

$$
\begin{gathered}
p_{x}=-61+u_{x} \cdot 118 \\
p_{x}=-61+u_{x} \cdot 105.5
\end{gathered}
$$

\section{Evaluation of the device's accuracy}

\begin{tabular}{|c|c|c|c|c|c|c|c|c|}
\hline \multirow{3}{*}{$\begin{array}{c}\text { Set } \\
\text { number }\end{array}$} & \multicolumn{2}{|c|}{ New Device } & \multicolumn{2}{|c|}{$\begin{array}{c}\text { Auscultatory } \\
\text { method }\end{array}$} & \multicolumn{2}{|c|}{ Method 1} & \multicolumn{2}{|c|}{ Method 2} \\
\hline & $\mathrm{Ud}_{\mathrm{d}}$ & $\mathrm{U}_{\mathrm{s}}$ & $\mathrm{p}_{\mathrm{d}}$ & $\mathrm{p}_{\mathrm{s}}$ & $\mathrm{p}_{\mathrm{d}}$ & $\mathrm{p}_{\mathrm{s}}$ & $\mathrm{p}_{\mathrm{d}}$ & $\mathrm{p}_{\mathrm{s}}$ \\
\hline & \multicolumn{2}{|c|}{ [V] } & \multicolumn{2}{|c|}{$[\mathrm{mmHg}]$} & \multicolumn{2}{|c|}{$[\mathrm{mmHg}]$} & \multicolumn{2}{|c|}{$[\mathrm{mmHg}]$} \\
\hline 98 & 1.22 & 1.66 & 75 & 120 & 81 & 130 & 74 & 120 \\
\hline 103 & 1.14 & 1.49 & 60 & 110 & 73 & 111 & 66 & 103 \\
\hline 116 & 1.24 & 1.55 & 72 & 125 & 84 & 118 & 76 & 109 \\
\hline 122 & 1.27 & 1.61 & 72 & 110 & 86 & 124 & 79 & 115 \\
\hline
\end{tabular}

After successful designation of calibration equations the second data set group was used for validation of both calibration equations. The pressure was calculated with use of both equations for comparison purposes depending only on new device's diastolic and systolic voltage values (Table 2).

Table 2. Obtained data sets and calculated coefficients.

Mean relative errors ( $\delta$ ) and standard deviations (s) were calculated for both methods.

Table 2. Calculated mean relative errors and standard deviations of both methods.

\begin{tabular}{|c|c|c|c|c|c|}
\hline \multirow{2}{*}{$\begin{array}{c}\text { Statistical } \\
\text { parameter }\end{array}$} & \multirow{2}{*}{ Unit } & \multicolumn{2}{|c|}{ Method 1 } & \multicolumn{2}{c|}{ Method 2 } \\
\cline { 3 - 6 } & & $\mathrm{p}_{\mathrm{d}}$ & $\mathrm{p}_{\mathrm{s}}$ & $\mathrm{p}_{\mathrm{d}}$ & $\mathrm{p}_{\mathrm{s}}$ \\
\hline$\delta$ & {$[\%]$} & 16.6 & 4.4 & 6.2 & -3.7 \\
\hline $\mathrm{s}$ & {$[\%]$} & 5.8 & 8.3 & 5.2 & 7.8 \\
\hline
\end{tabular}




\section{Conclusions}

The developed device for measurement and registration of blood pressure waveform, despite the excellent measuring parameters exhibited during evaluation in laboratory conditions (on the artificial artery), did not give accurate results during tests on volunteers. The maximum error exceeded $20 \%$, the mean error equalled $12 \%$ for diastolic and $10 \%$ for systolic blood pressure. Herein, the attempt of preliminary improvement of the device's accuracy was taken with use of two calibration methods - standard method 1 based on mean linear equation's coefficients and the modification of standard method 2 with one of the coefficients corrected based on mathematical model of the device. Therefore the research was conducted on volunteers in accordance to adequate protocol [6] to acquire data from two methods - new method and auscultatory method. After successful data acquisition two calibration equations were designated and validated. It was found that the corrected equation (6) was indeed more accurate than the standard calibration equation (5). The mean errors for the blood pressure calculated based on equation (6) were consecutively $6.2 \%$ and $-3.7 \%$ for diastolic and systolic pressure. These results do not meet the requirements for human blood pressure measuring devices. However, it is worth mentioning that in comparison with the currently available devices (non-invasive), the new device can register the blood pressure waveform which is a very valuable diagnostic object for evaluation of cardiovascular system condition. Further research may concern development of a more accurate corrected equation based on a mathematical model of a wrist with the radial artery, which is the most suitable location for taking measurements with the new device.

\section{References}

1. M. Ward, J. A. Langton, Continuing Education in Anaesthesia, Critical Care \& Pain 7, 4 (2007)

2. G. Ogedegbe, T. Pickering, Cardiol Clin. 28, 4 (2010)

3. G. A. van Montfrans, Blood Pressure Monitoring, 6, 6 (2001)

4. A. Bur, M. M. Hirschl, H. Gerkner, E. Oschatz, J. Kofler, C. Woiserschläger, A. N. Laggner, Crit Care Med. 28, 2 (2000)

5. K. Tomczuk, M. Werszko, J. Z. Sąsiadek, J. Kosek, W.Berny, A. Weiser, J. Feder-Kubis, Review of Scientific Instruments 84, 095003 (2013)

6. International Protocol for Validation of Blood Pressure Measuring Devices in Adults, Blood Press Monit. 15, 3 (2010)

7. Werszko M., Tomczuk K., Jędrusyna A.; Pneumatic sensors for blood pressure measurement; International Conference Mechatronics; 2004; Warszawa; 23-25.09.2004 